\title{
Changes of Colon Flora and Antitoxic Effect Induced by Zearalenone in Mice
}

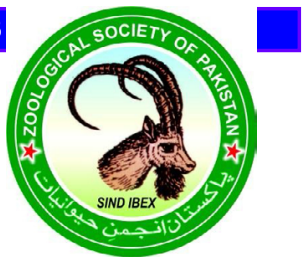

\author{
Jing Zhang ${ }^{1,2}$, Xin Wang ${ }^{2}$, Yun Zhao' ${ }^{2}$, Yongcheng $\mathrm{Jin}^{2}$, Yongfeng Zhou ${ }^{2}$, Junmei Wang ${ }^{2}$, \\ Yurong $\mathrm{Fu}^{2}$, Rui Wang ${ }^{2}$, Ruihua $\mathrm{Li}^{2}$, Hengtong $\mathrm{Fang}^{2}$ and ${\mathrm{Hao} \mathrm{Yu}^{2}, * *}^{2}$ \\ ${ }^{1}$ Key Laboratory of Zoonosis Research, Ministry of Education, Jilin University, \\ Changchun 130062, China \\ ${ }^{2}$ College of Animal Science, Jilin University, Changchun 130062, China
}

Hengtong Fang and Hao Yu contributed equally to this article.

\begin{abstract}
A B S T RA C T
Although mounting evidence has shown that mycotoxins can cause intestinal dysfunction and mucosal immune disorders, the effect of zearalenone (ZEA) on intestinal mucosal function remains controversial. We aimed to explore the effects of short-term ZEA exposure on the mucosal barrier function in the colon. We found that the morphology of colonic mucosa in mice was normal after ZEA was administered by gavage for one week, the mRNA expression levels of mucosal Mucin-1, Mucin-2, regenerating isletderived protein 3 gamma (Reg3 $\gamma$ ), and tumor necrosis factor (TNF) were significantly downregulated. The mRNA expression levels of mucosal $\beta$-defensin, regenerating islet-derived protein 3 alpha (Reg3a), regenerating islet-derived protein 3 beta $(\operatorname{Reg} 3 \beta)$ and secretory immunoglobulin A (sIgA) levels were significantly increased. The interleukin-1beta (IL-1 $\beta$ ) level was decreased. 16s RNA sequencing further indicated that the colonic microflora had changed, especially the Lactobacillus had increased significantly. Our study showed that short-term ZEA exposure led to resistive increase of colonic probiotics in mice, especially the increase of Lactobacillus can reduce intestinal mucosal immunotoxicity.
\end{abstract}

\begin{tabular}{l} 
Article Information \\
\hline Received 15 January 2019 \\
Revised 12 May 2019 \\
Accepted 25 September 2019 \\
Available online 17 April 2020 \\
Authors' Contribution \\
\hline HY, HF, XW and JZ designed the \\
study. JZ, XW, YZ, YJ and YZ, \\
performed the experimental work. \\
HF and HY analyzed the data. JW, \\
YF, RW, RL provided some help for \\
this experiment. HY and HF wrote \\
the article. \\
Key words \\
\hline Zearalenone, Intestinal microflora, \\
Lactobacillus, Mucosal immunity, \\
Antitoxic effect
\end{tabular}

Article Information

Revised 12 May 2019

Accepted 25 September 2019

Authors' Contribution

$\mathrm{HY}, \mathrm{HF}, \mathrm{XW}$ and $\mathrm{JZ}$ designed the study. JZ, XW, YZ, YJ and YZ performed the experimental work. $\mathrm{HF}$ and $\mathrm{HY}$ analyzed the data. JW,

F, RW, RL provided some help for this experiment. HY and HF wrot

Zearalenone, Intestinal microflora,

Antitoxic effect

\section{INTRODUCTION}

A ccording to previous reports, zearalenone (ZEA) is one of the most threatening environmental toxins in the world and it was associated with many fungal diseases in farm animals (Fink-Gremmels and Malekinejad, 2007). Nowadays, we often concern that ZEA induces reproductive toxicity in mammals. There are few studies on the toxic effects of ZEA on intestinal mucosal barriers in animal or human. Studies have revealed that ZEA impairs the small intestinal mucosal barrier (Liu et al., 2014), and we have previously demonstrated that ZEA not only disrupts the jejunal microecological balance but also causes inflammation of the jejunal mucosa (Wang et $a l ., 2018$ ). But we cannot ignore that colon contains large number of intestinal microbes and its microecological stability is more vulnerable to interference from external substances. Increasing evidence suggests that the incidence of colitis and colon cancer is closely related to environmental pollutants, especially mycotoxins (Wild and Gong, 2010; Seonghwan et al., 2010; Maresca and Fantini, 2010). Therefore, it is necessary to investigate the effects of ZEA exposure on the colonic mucosal barrier

\footnotetext{
* Corresponding author: yu_hao@jlu.edu.cn 0030-9923/2020/0004-1555 \$ $\overline{9} .00 / 0$

Copyright 2020 Zoological Society of Pakistan
}

and microecological stability. As we all know, the intestinal mucosa is the first line of defense of the intestinal barrier. Notably, the intestinal epithelial cells, intestinal flora, mucus, sIgA and antimicrobial peptides (AMPs) in the intestinal mucosa constitute the "firewalls" of intestinal mucosal immunity, which protect the body from pathogenic bacteria, virus and environmental pollutants (Macpherson et al., 2009). Recent studies have shown that most mycotoxins can induce intestinal epithelial cell inflammation by destroying intestinal mucosal barrier (Seonghwan et al., 2010). Indeed, The intestinal flora plays a role in regulating intestinal immunity, and it acts as an adjuvant to the entire immune system (Molloy et al., 2012; Belkaid and Hand, 2014). However, there are few studies on the toxic effects of ZEA on the intestinal microflora and colonic mucosal barrier in mammals, and its mechanism is still unclear (Gajęcka et al., 2016).

Therefore, we systematically investigated the effects of ZEA on colonic microorganisms and mucosal barriers in mice by $16 \mathrm{~S}$ rRNA sequencing technology, and explored the potential molecular mechanisms of reduced immunotoxicity, which provided theoretical basis for prevention and treatment of ZEA exposure.

\section{MATERIALS AND METHODS}

ZEA (purity $>98 \%$ ) was purchased from SigmaAldrich (St Louis, MO, USA). It was dissolved in ethanol 
and made into $100 \mathrm{mg} / \mathrm{ml}$. Then the storage solution was dissolved in olive oil at $10 \mathrm{mg} / \mathrm{ml}$ products before use.

Twenty-four immature male BALB/C mice (Laboratory Animal Center of Jilin University, China), were individually divided into two groups, each group had three replicates, each containing four mice. After a weekend for a trial period, all mice were reared under standard test conditions, and were fed by basal diet (Liaoning Changsheng Biological Company) and sterile drinking water. The recommended dose of ZEA induces a physiological disorder in mice of $20 \mathrm{mg} /(\mathrm{kg}$. bw) $\left(\sim 1 \% \mathrm{LD}_{50}\right)$ (Zinedine et al., 2007). We adopted this recommended dose and conducted many tests on mice, we found it was indeed effective. Therefore, the experimental group (ZEA group) was administered ZEA by oral gavage at a dosage of $20 \mathrm{mg} / \mathrm{kg}$ body weight $\left(\sim 1 \% \mathrm{LD}_{50}\right)$ once daily for one week, and the control group (CON group) was given an equal volume of vector. All experiments were approved by the Institutional Animal Care and Use Committee of Jilin University.

At the end of the experiment, the mice were sacrificed by cervical dislocation. The colon was separated, and the intermediate segment (1-2 cm long) was taken and washed away with saline, and the tissue was fixed with $4 \%$ polyoxymethylene. The remaining colon and feces were carefully collected, quickly placed in liquid nitrogen, and then transferred to $-80^{\circ} \mathrm{C}$.

\section{Intestinal mucosal morphology analysis}

The intestinal tissues were fixed with $4 \%$ polyoxymethylene and embedded in petrolin, then sectioned into 2-3 $\mu \mathrm{m}$ sections. The sections were stained with hematoxylin first, counterstained with eosin, and finally observed under an optical microscope.

\section{Composition and diversity of the bacterial communities}

Total genomic bacterial DNA was extracted from the feces of colon in BALB/c mice. Then the qualified genomic DNA was used for 16S-rDNA V3V4 region PCR amplification. The primer was 341F (5', ACTCCTACGGGAGGCAGCAG-3') and 806R (5' GGACTACHVGGGTWTCTAAT-3'). All the procedures were performed according to the manufacturer's protocol (BGI, Shengzhen, China). Two hypervariable regions of $16 \mathrm{~S}$ rDNA, the V3 and V4 regions, were used to identify the vast majority of the bacteria based on the $16 \mathrm{~S}$ rDNA sequencing. The qualified PCR products were purified by $16 \mathrm{~S}$ V3-V4 amplification, and the DNA library was constructed. The qualified DNA library was sequenced using an Illumina MiSeq $2 * 300$, and information was acquired for the bioinformatic analysis.

The raw data were filtered to eliminate adapter pollution and low quality to obtain clean reads. Then, the paired-end reads with overlapping regions were merged to raw tags with FLASH software (v1.2.11) (Magoč and Salzberg, 2011). Paired-end reads without overlaps were removed. Following removal of the primer sequences, the forward and reverse amplification primers were mapped to the two ends of the tags. The tags were clustered to the operational taxonomic unit (OUT) at 97\% sequence similarity by scripts using the software USEARCH (v7.0.1090) (RC, 2013). The PCR-generated chimaera from the OTU representation sequence was removed, and effective tags were obtained by UCHIME software (v4.2.40) (Edgar et al., 2011). The database used for species annotation was Greengene (V201305) (Desantis et al., 2006).

Determination of the relative expression of $m R N A$ in intestinal mucosal barrie by real-time PCR

Total RNA was extracted from colon using TriPure (Roche, Basel, Switzerland) and the cDNA was synthesized using Reverse Transcription System (Promega, Madison, USA) and SYBR Green Mix Kit (Trans, Beijing, China) was used for real-time PCR. Primer design as shown in Supplementary Table I.

\section{Detection of SIgA in the colon}

Equal amounts of colonic feces were dissolved with $0.01 \mathrm{M} \mathrm{PBS}$, and broken down at low temperature, centrifuged for $10 \mathrm{~min}$ at $3000 \mathrm{r}$, then the supernatant was collected. The total protein concentration of the feces was detected by BCA. Then the absorbance was detected at 562 $\mathrm{nm}$ according to the instructions of mouse sIgA ELISA kit (eBioscience, California, USA), and the sIgA content of feces was calculated $(\mathrm{ng} / \mathrm{ml})$.

\section{Detection of cytokine in the colon}

Colon was homogenized with $0.01 \mathrm{M}$ PBS to make $10 \%$ tissue homogenate. Then at low temperature, the homogenate was centrifuged for $10 \mathrm{~min}$ at $3000 \mathrm{r}$. The supernatant was used for estimation of total protein according to the instructions of IL-1 $\beta$, IL-10, and TNF (Naniing Jiancheng Bioengineering Institute, Nanjing, China). The cytokine levels were calculated as ng/g prot.

\section{Statistical analysis}

All the data were analysed using SPSS statistical software (version 20.0). The variation between the CON group and the ZEA group was analysed by a t-test, and the results are expressed as the mean \pm SEM. The differences were judged as statistically significant at $\mathrm{P}$ values $<0.05$.

\section{RESULTS}

\section{Effect of ZEA on mucosal morphology}

To objectively assess the effects of ZEA on intestinal 
mucosa, the histological sections of colon mucosal morphology was observed, as shown in Figures 1A, $1 \mathrm{~B}$. We found that there was no significant pathological change between ZEA group and control group, but the intestinal cavity of the ZEA group became significantly larger (Fig. 1C).
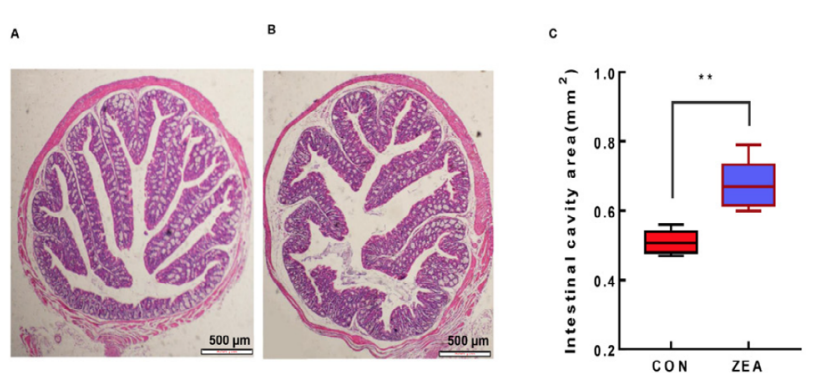

Fig. 1. The effect of ZEA on the intestinal mucosal morphology of the colon in BALB/C mice. A: The colon of the CON group. B: The colon of the ZEA group. C: The area of intestinal cavity. $* *$ : $\mathrm{P}$ value $<0.01$.

Table I. Effect of ZEA on bacterial community diversity in the colonic digesta.

\begin{tabular}{llllll}
\hline $\begin{array}{l}\text { Sample } \\
\text { name }\end{array}$ & $\begin{array}{l}\text { Tag } \\
\text { number }\end{array}$ & $\begin{array}{l}\text { OTU } \\
\text { number }\end{array}$ & Length(bp) & ace & simpson \\
\hline CON & 25379 & 286 & 415 & 304.4 & 0.057 \\
ZEA & 24024 & 295 & 416 & 318.0 & 0.035 \\
\hline
\end{tabular}

ZEA changes the balance of the intestinal flora

In order to study the effect of ZEA on intestine microflora in the mice, a total of 148209 effective tags were obtained by $16 \mathrm{~S}-\mathrm{rRNA}$ sequencing, with an average of 24702 effective tags for each sample and an average length of $416 \mathrm{bp}$ (Table I). Using the ACE index (Table I) to assess bacterial richness, short-term ZEA exposure led to a tendency to increase the richness of the colonic microflora. At the same time, the Simpson index was used to evaluate changes in the intestinal microbial diversity (Table I). In the ZEA group, the bacterial diversity was almost similar to that of the CON group, but the diversity of the colonic microflora showed a tendency to increase. At $97 \%$ similarity, the colonic bacterial community of the CON and ZEA groups shared 325 OTUs (Fig. 2A). The flattening of the rarefaction curve based on the values of observed species (Fig. 2B) indicated that our data volume covered all species of the community in the colon samples. At phylum level (Fig. 2C), compared with the CON group, the abundance of Proteobacteria in the ZEA group was reduced more than 2 -fold $(\mathrm{P}<0.001)$. The abundance of Actinobacteria in the ZEA group was increased $(\mathrm{P}<$
0.01). At genus levels (Fig. 2C), Lachnoclostridium and Alloprevotella were highly abundant. Compared to the CON group, ZEA reduced Lachnoclostridium from 16.7\% to $9.8 \%(\mathrm{P}>0.05)$ and reduced Alloprevotella from $9.6 \%$ to $4.9 \%(\mathrm{P}>0.05)$. The abundance of Desulfovibrio was reduced from $3.1 \%$ to $1.5 \%(\mathrm{P}>0.05)$. Compared with $\mathrm{CON}$ group, the abundance of Anaeroplasma, Flavonifractor, Helicobacter, Prevotella and Ruminiclostridium in the ZEA group was decreased $(\mathrm{P}>0.05)$. Interestingly, the Lactobacillus was upregulated more than 4-fold $(\mathrm{P}<$ 0.05). In addition, we found that short-term ZEA exposure led the emergence of Akkermansia in colon.

\section{Effect of ZEA on intestinal mucosal barrier in mice}

We further detected the effect of ZEA on colonic mucosal barrier. We examined the mRNA expression of key genes that play the first line of defense in the colonic mucosa (Fig. 3). The mRNA expression of Mucin 1, Mucin 2 , and $\operatorname{Reg} 3 \gamma$ were significantly downregulated by ZEA exposure. Interestingly, ZEA increased mRNA expression of $\beta$-defensin, $\operatorname{Reg} 3 \alpha$, and Reg $3 \beta$. In addition, the mRNA expression of inflammatory cytokines IL- $1 \beta$ and TNF were remarkably downregulated after ZEA exposure. There was no significant change in the mRNA level of IL- 10 .

\section{The effect of ZEA on intestinal mucosal immunity}

To assess the effect of ZEA on intestinal mucosal immunity, we firstly detect the faecal sIgA levels in the colon. The results showed that short-term ZEA exposure induced the mucosal $\operatorname{sigA}(\mathrm{P}<0.001)$ and the faecal $\operatorname{IgA}$ in the ZEA group increased more than 3-fold compared to that in the CON group (Fig. 4A). At the same time, we investigated the effects of ZEA on cytokine secretion involved in the inflammatory response in the colon. Interestingly, ZEA inhibited the secretion of IL-1 $\beta$ in the colon, but there was no change in TNF and IL-10 levels (Fig. 4B).

\section{DISCUSSION}

Mycotoxin is a class of environmental pollutant that easily causes an intestinal ecological imbalance and induces some intestinal diseases (Maresca and Fantini, 2010; Choung and Iii, 2011; Compare and Nardone, 2014). We found that the structure of colonic microbial communities was altered by short-term ZEA exposure. Changes in some of the dominant bacteria in the gut indicated that our ZEA exposure induced disturbances in the intestinal microecology. We found that Proteobacteria which are associated with inflammation was decreased (Carvalho et al., 2012). At the same time, Lachnoclostridium and Alloprevotella, Desulfovibrio, 


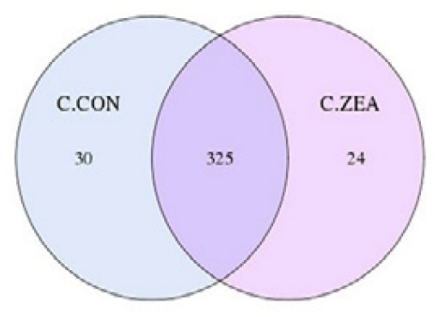

A

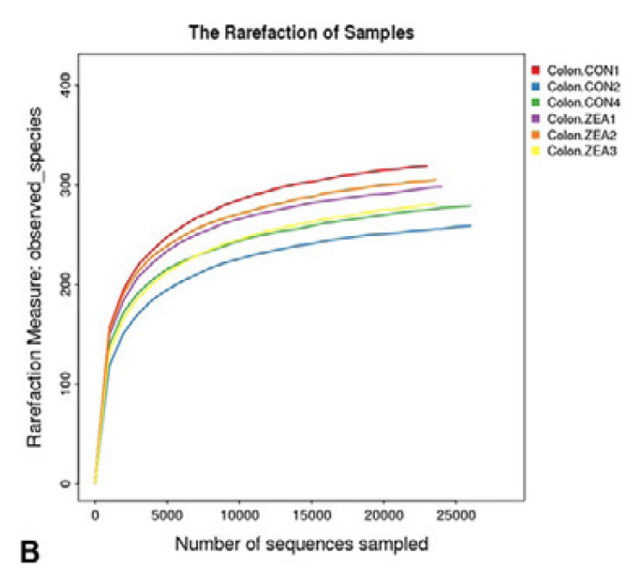

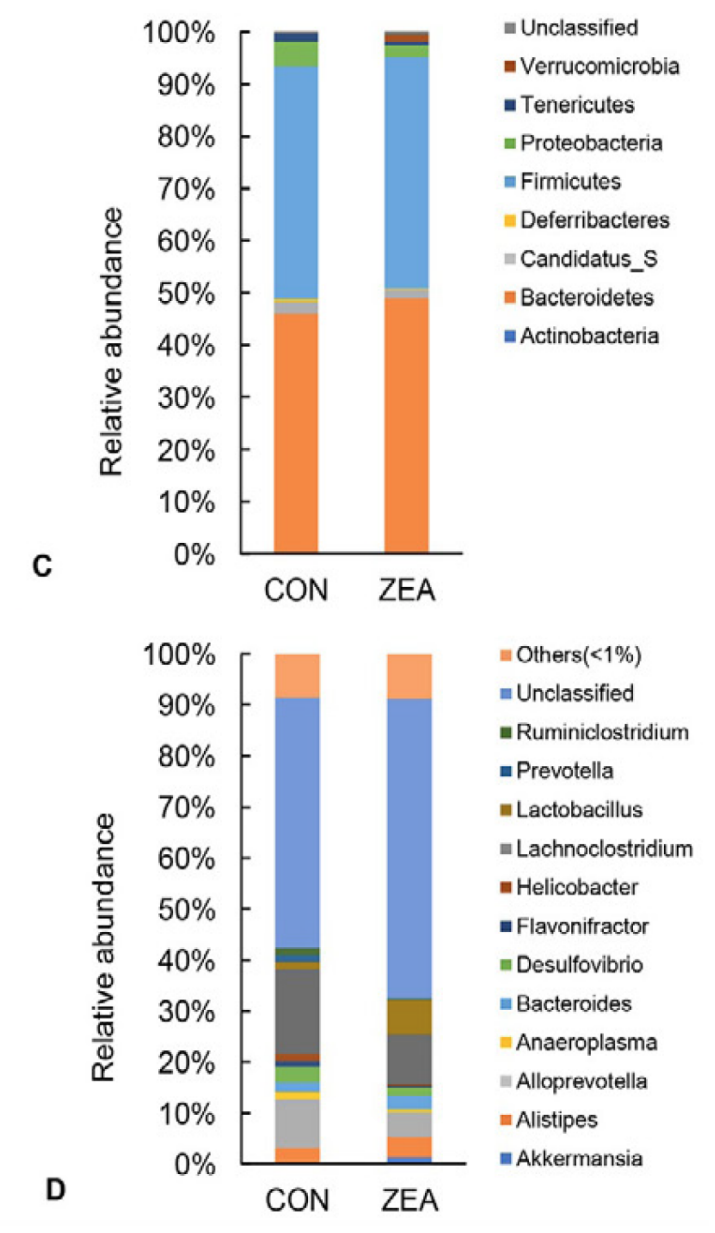

Fig. 2. Effect of ZEA on the intestinal microflora. (A), comparison of OTU. (B), rarefaction curves based on the observed species values. (C), relative abundance of different bacterial phylum. (D), relative abundance of different bacterial genus.

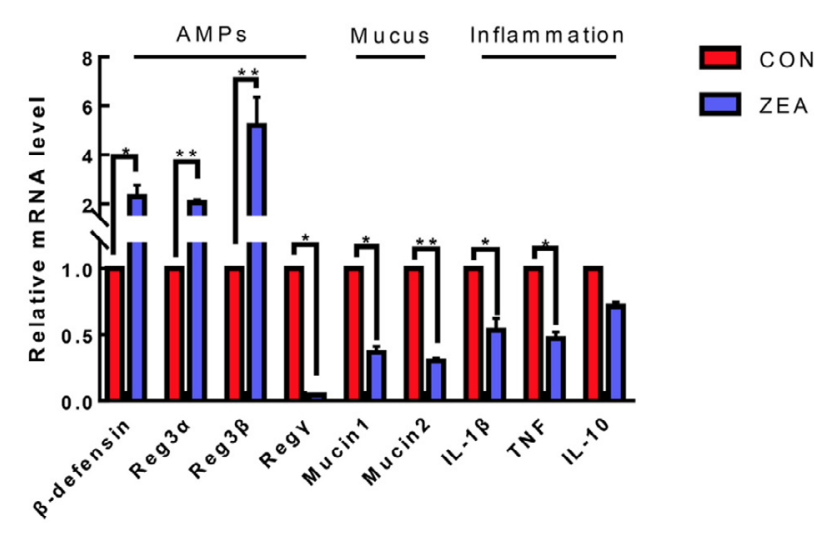

Fig. 3. Changes in the immune gene expression in the colonic mucosa of the mice. All the data are expressed as the means \pm SEM. *: P value $<0.05, * *:$ P value $<0.01$.
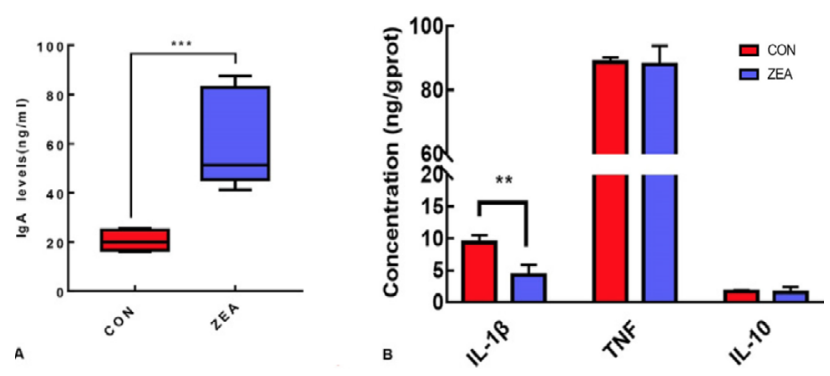

Fig. 4. (A), Changes in the sIgA levels in the colonic faeces. (B), Changes in the cytokines in the colon. All the data are expressed as the means \pm SEM. $* *$ : $\mathrm{P}$ value $<0.01$, $* * *$ : $\mathrm{P}$ value $<0.001$

Anaeroplasma, Flavonifractor, Helicobacter, Prevotella and Ruminiclostridium were decreased. Previous studies have shown that Clostridium leptum, Lachnospiraceae, and Prevotellaceae were reduced in inflammatory bowel 
disease patients (Swidsinski et al., 2002). Changes in these micrbiotes suggest instability of the intestinal flora or sensitivity of the intestines. It is worth noting that ZEA increased the Lactobacillus. Piotrowska et al. (2014) argued that long-term exposure to low doses of ZEA caused an increase in the Lactobacillus in the colon, but the change was not significant. Reddy et al. (2018) found that the ZEA-contaminated feed significantly affected the colon microbiota, especially Lactobacillus, which was increased by $2.7 \%$. They speculate that Lactobacillus plays a major role in detoxification of these mycotoxins. Indeed, Lactobacillus have been evaluated in studies in animals and humans with respect to antibiotic-associated diarrhoea, travellers' diarrhoea, pediatric diarrhoea, inflammatory bowel disease, and irritable bowel syndrome. Lactobacillus is not only beneficial to intestinal health (Sonnenburg et al., 2005) but also combined with mycotoxins to remove mycotoxins (Elnezami et al., 2002). The decrease of Proteobacteria and the increase of Lactobacillus seem to indicate that damage to the colon by ZEA has been controlled, as evidenced by normal colon morphology. We suspect that the increase of the intestinal cavity area may also be due to the changes in intestinal flora.

Further studies found that ZEA exposure significantly reduced the mRNA expression of Mucin 1, Mucin 2 and Reg3 $\gamma$ in colonic mucosa. Current studies suggest that ZEA increases the secretion of mucin in Caco-2/HT29MTX cells (Wan et al., 2014). Reg3 $\gamma$ and mucins are used to prevent microbial contact with the epithelial layer (Turner, 2009; Sanchez et al., 2014; Stefania et al., 2015). The decrease of these mRNA expressions indicates the decrease of mucosal defense. Interestingly, ZEA increased the mRNA expression of colonic mucosal $\beta$-defensin, $\operatorname{Reg} 3 \alpha$ and $\operatorname{Reg} 3 \beta$. In addition, the $\operatorname{sg} A$ in the colon was also increased. Indeed, $\beta$-defensin Reg $3 \alpha / \beta$ and $\operatorname{sIg} A$ play an essential role in the immune defense of the intestinal mucosa (Wang et al., 2015).

ZEA has been described as either an inductor or a suppressor of pro-inflammatory cytokines (Salah-Abbès et al., 2010; Marin et al., 2011). Our study found that ZEA exposure inhibited the IL-1 $\beta$ and the mRNA expression of TNF was downregulated. Fan et al. (2017) suggested that ZEA leads to increased levels of IL- $1 \beta$ in colon tissue of mice. It contradicts our findings. However, Liu et al. (2014) revealed that different doses of ZEA showed a downward trend of IL-1 $\beta$ and TNF in the jejunum of rats. Braicu et al. (2016) found that exposure to ZEA caused a decrease in the IL-1 $\beta$ and TNF in animal which is still waiting for us to further study.

Current study suggests that ZEA is absorbed through the small intestine and some of it is degraded through the circulation in the liver and intestine (Fan et al., 2017) while some combines with intestinal microbes. Eventually, only a small amount of ZEA and its degradation products act on the large intestine (Kollarczik et al., 2010). On one hand, a low dose of ZEA is considered to be immunosuppressant (Bondy and Pestka, 2000) and may inhibit the inflammatory response. On the other hand, the increase of colonic Lactobacillus only remove mycotoxins by combining with mycotoxins but also regulate intestinal mucosal immunity by producing short chain fatty acid (SCFA) (Elnezami et al., 2002; Martín et al., 2013). Thereby mucosal damage is alleviated to some extent. But the specific reasons are still waiting to be explored.

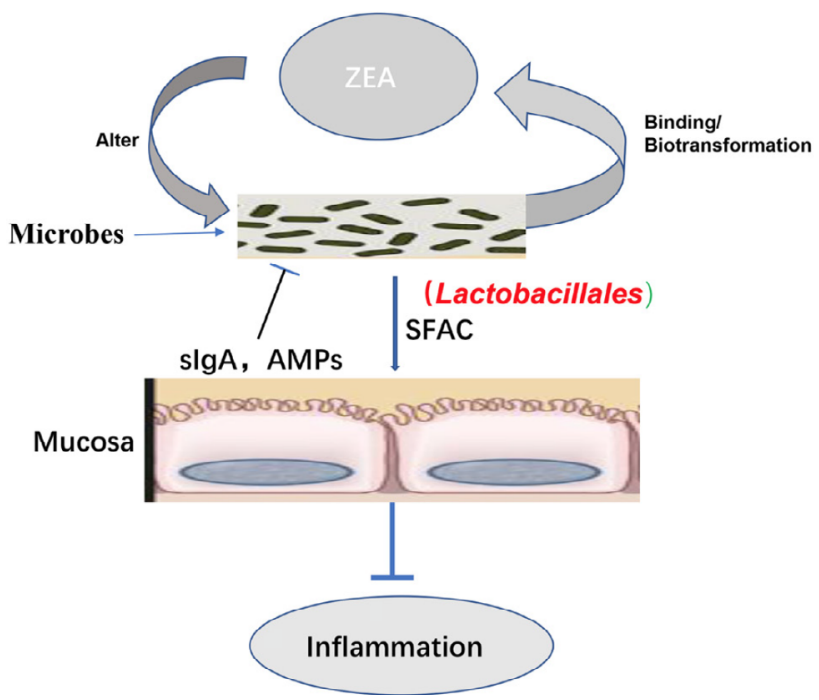

Fig. 5. Schematic diagram of ZEA acting on colonic mucosa.

\section{CONCLUSION}

Our study found thatZEA enhanced mucosal immunity in the colon and decreased the colonic Proteobacteria. It is worth noting that the intestinal Lactobacillus was increased. We believe that low doses of ZEA enter the colon and stimulate the growth of beneficial bacteria in the colon (Fig. 5), which indirectly activates the mucosal defense system. Ultimately the toxicity of ZEA to the intestinal mucosa was alleviated. All of this credit comes from the special role of Lactobacillus, which play a key role in regulating intestinal mucosal immunity and promoting colon health. Thus, the colonic mucosa can protect against a certain degree of toxins such as ZEA. This appears to provide us with a new way of thinking about controlling mycotoxins. We believe that intestinal Lactobacillus play an essential role in the intestinal mucosal immune defense against environmental toxins such as mycotoxins. 


\section{ACKNOWLEDGEMENTS}

This study was financially supported by the Jilin Scientific and Technological Development Program (20170307018NY, 20190301034NY); the Special Project of the Province-University Co-constructing Program of Jilin Province (SXGJXX2017-4); the central finance forestry science and technology promotion demonstration fund project and the National Key Research and Development Program of China Stem Cell and Translational Research (2017YFA0105101); the Open Projects of Northeast Agricultural University/Scientific Observing and Experimental Station of Animal Nutrition and Feed Science in Northeast, Ministry of Agriculture, P.R. China; and the Jilin Modern Agricultural Technology Demonstration and Extension Project.

\section{Supplementary material}

There is supplementary material associated with this article. Access the material online at: https://dx.doi. org/10.17582/journal.pjz/20190115060153

\section{Statement of conflict of interest}

The authors declare that there are no conflicts of interest.

\section{REFERENCES}

Belkaid, Y. and Hand. T.W., 2014. Role of the microbiota in immunity and inflammation. Cell, 157: 121-141. https://doi.org/10.1016/j.cell.2014.03.011

Bondy, G.S. and Pestka. J.J., 2000. Immunomodulation by fungal toxins. J. Toxicol. environ. Hlth. B. Crit. Rev., 3: 109-143. https://doi. org/10.1080/109374000281113

Braicu, C., Cojocneanupetric, R., Jurj, A., Gulei, D., Taranu, I., Gras, A.M., Marin, D.E. and Berindanneagoe, I., 2016. Microarray based gene expression analysis of sus scrofa duodenum exposed to zearalenone: significance to human health. $B M C$ Genom., 17:646. https://doi.org/10.1186/s12864016-2984-8

Carvalho, F.A., Koren, O., Goodrich, J.K., Johansson, M.E.V., Nalbantoglu, I., Aitken, J.D., Su, Y., Chassaing, B., Walters, W.A. and González. A., 2012. Transient inability to manage proteobacteria promotes chronic gut inflammation in TLR5deficient mice. Cell Host Microbe, 12: 139-152. https://doi.org/10.1016/j.chom.2012.07.004

Choung, R.S. and Iii, G.R.L., 2011. Epidemiology of IBS. Gastroenterol. Clin. N., 40: 1-10. https://doi. org/10.1016/j.gtc.2010.12.006
Compare, D., and Nardone, G., 2014. The role of gut microbiota in the pathogenesis and management of allergic diseases. Eur. Rev. Med. Pharmacol. Sci., 18: 11-17.

Desantis, T. Z., Hugenholtz, P., Larsen, N., Rojas, M., Brodie, E.L., Keller, K., Huber, T., Dalevi, D., $\mathrm{Hu}$, P. and Andersen, G.L., 2006. Greengenes, a chimera-checked 16S rRNA gene database and workbench compatible with ARB. Appl. environ. Microbiol., 72: 5069-5072. https://doi.org/10.1128/ AEM.03006-05

Edgar, R. C., Haas, B. J., Clemente, J. C., Christopher, Q. and Rob, K., 2011. UCHIME improves sensitivity and speed of chimera detection. Bioinformatics, 27 : 2194-2200. https://doi.org/10.1093/bioinformatics/ btr381

Edgar, R.C., 2013. UPARSE: highly accurate OTU sequences from microbial amplicon reads. Nat. Methods, 10: 996-998. https://doi.org/10.1038/ nmeth.2604

Elnezami, H.S., Chrevatidis, A., Auriola, S., Salminen, S. and Mykkänen, H., 2002. Removal of common Fusarium toxins in vitro by strains of Lactobacillus and Propionibacterium. Fd.Addit. Contam., 19:680686. https://doi.org/10.1080/02652030210134236

Fan, P., Song, P., Li, L., Chang, H., Chen, J., Yang, W., Qiao, S., Wu, G., Zhang, G. and Xi, M., 2017. Roles of biogenic amines in intestinal signaling. Curr. Protein Pept. Sci., 18: 532-540. https://doi.or $\mathrm{g} / 10.2174 / 1389203717666160627073048$

Fan, W., Lv, Y., Ren, S., Shao, M., Shen, T., Huang, K., Zhou, J., Yan, L. and Song. S., 2017b. Zearalenone (ZEA)-induced intestinal inflammation is mediated by the NLRP3 inflammasome. Chemosphere, 190: 272-279. https://doi.org/10.1016/j. chemosphere.2017.09.145

Fink-Gremmels, J. and Malekinejad. H., 2007. Clinical effects and biochemical mechanisms associated with exposure to the mycoestrogen zearalenone. Anim. Feed Sci. Tech., 137: 326-341. https://doi. org/10.1016/j.anifeedsci.2007.06.008

Gajęcka, M., Zielonka, Ł. and Gajęcki. M., 2016. Activity of zearalenone in the porcine intestinal tract. Molecules, 22: 18. https://doi.org/10.3390/ molecules22010018

Kollarczik, B., Gareis, M. and Hanelt, M., 2010. In vitro transformation of the fusarium mycotoxins deoxynivalenol and zearalenone by the normal gut microflora of pigs. Nat. Toxins, 2: 105-110. https:// doi.org/10.1002/nt.2620020303

Liu, M., Gao, R., Meng, Q., Zhang, Y., Bi, C. and Shan. A., 2014. Toxic effects of maternal zearalenone 
exposure on intestinal oxidative stress, barrier function, immunological and morphological changes in rats. PLoS One, 9: e106412. https://doi. org/10.1371/journal.pone.0106412

Macpherson, A.J., Slack, E., Geuking, M.B. and Mccoy, K.D., 2009. The mucosal firewalls against commensal intestinal microbes. Semin. Immunopathol., 31: 145-149. https://doi. org/10.1007/s00281-009-0174-3

Magoč, T. and Salzberg, S.L., 2011. FLASH: fast length adjustment of short reads to improve genome assemblies. Bioinformatics, 27: 2957-2963. https:// doi.org/10.1093/bioinformatics/btr507

Maresca, M. and Fantini, J., 2010. Some food-associated mycotoxins as potential risk factors in humans predisposed to chronic intestinal inflammatory diseases. Toxicon, 56: 282-294. https://doi. org/10.1016/j.toxicon.2010.04.016

Marin, D.E., Taranu, I., Burlacu, R., Manda, G., Motiu, M., Neagoe, I., Dragomir, C., Stancu, M. and Calin. L., 2011. Effects of zearalenone and its derivatives on porcine immune response. Toxicology in vitro., 25: 1981-1988. https://doi.org/10.1016/j. tiv.2011.06.022

Martín, R., Miquel, S., Ulmer, J., Kechaou, N., Langella, P., and Bermúdezhumarán, L.G., 2013. Role of commensal and probiotic bacteria in human health: a focus on inflammatory bowel disease. Microbe Cell Fact., 12: 71-71. https://doi.org/10.1186/14752859-12-71

Molloy, M.J., Bouladoux, N. and Belkaid, Y., 2012. Intestinal microbiota: Shaping local and systemic immune responses. Semin. Immunol., 24: 58-66. https://doi.org/10.1016/j.smim.2011.11.008

Muriel, D., Vlieg, J.E.T.H., 2015. Fate, activity, and impact of ingested bacteria within the human gut microbiota. Trends Microbiol., 23: 354-366. https:// doi.org/10.1016/j.tim.2015.03.002

Piotrowska, M., Śliżewska, K., Nowak, A., Zielonka, Ł., Żakowska, Z., Gajęcka, M. and Gajęcki, M., 2014. The effect of experimental fusarium mycotoxicosis on microbiota diversity in porcine ascending colon contents. Toxins, 6: 2064-2081. https://doi. org/10.3390/toxins6072064

Reddy, K.E., Jeong, J.Y., Song, J., Lee, Y., Lee, H.J., Kim, D.W., Jung, H.J., Kim, K.H., Kim, M., Oh, Y.K., Lee, S.D. and Kim, M., 2018. Colon microbiome of pigs fed diet contaminated with commercial purified deoxynivalenol and zearalenone. Toxins, 10: 347 . https://doi.org/10.3390/toxins 10090347

Richards, J.D., Lange, J.G.F.M.D., 2005. The gastrointestinal microbiota and its role in monogastric nutrition and health with an emphasis on pigs: current understanding, possible modulations, and new technologies for ecological studies. Can. J. Anim. Sci., 85: 421-435. https://doi. org/10.4141/A05-049

Salah-Abbès, J.B., Abbès, S., Houas, Z., Abdel-Wahhab, M.A. and Oueslati, R., 2010. Zearalenone induces immunotoxicity in mice: Possible protective effects of radish extract (raphanus sativus). J. Pharm. Pharmacol., 60: 761-770. https://doi.org/10.1211/ jpp.60.6.0012

Sanchez de Medina, F., Romero-Calvo, I., Mascaraque, C. and Martinez-Augustin. O., 2014. Intestinal inflammation and mucosal barrier function. Inflamm. Bowel Dis., 20: 2394-2404. https://doi. org/10.1097/MIB.0000000000000204

Seonghwan, P., Hyejin, C., Yang, H., Keehun, D., Juil, K. and Yuseok, M., 2010. Repression of peroxisome proliferator-activated receptor $\gamma$ by mucosal ribotoxic insult-activated CCAAT/ enhancer-binding protein homologous protein. $J$. Immunol., 185: 5522-5530. https://doi.org/10.4049/ jimmunol.1001315

Sonnenburg, Justin, L., Xu, J., Leip, D., Chen, C.H., Westover, B.P., Weatherford, J., Buhler, J.D. and Gordon, J.I., 2005. Glycan foraging in vivo by an intestine-adapted bacterial symbiont. Science, 307: 1955-1959. https://doi.org/10.1126/ science. 1109051

Stefania, D.S., Elisabetta, C., Mauro, M., Emilio, J. and Marcello, C., 2015. Nutritional keys for intestinal barrier modulation. Front. Immunol., 6: 612. https://doi.org/10.3389/fimmu.2015.00612

Swidsinski, A., Ladhoff, A., Pernthaler, A., Swidsinski, S., Loening-Baucke, V., Ortner, M., Weber, J., Hoffmann, U., Schreiber, S. and Dietel, M., 2002. Mucosal flora in inflammatory bowel disease. Gastroent. Hepat., 122: 44-54. https://doi. org/10.1053/gast.2002.30294

Turner, J.R., 2009. Intestinal mucosal barrier function in health and disease. Nat. Rev. Immunol., 9: 799-809. https://doi.org/10.1038/nri2653

Wan, L.Y.M., Allen, K.J., Turner, P.C. and Elnezami. H., 2014. Modulation of mucin mRNA (muc5ac and muc5b) expression and protein production and secretion in caco-2/ht29-mtx co-cultures following exposure to individual and combined fusarium mycotoxins. Toxicol. Sci., 139: 83-98. https://doi. org/10.1093/toxsci/kfu019

Wang, J., Tang, H., Zhang, C., Zhao, Y., Derrien, M., Rocher, E., van-Hylckama, J., Strissel, K., Zhao, L., Obin, M. and Shen, J., 2015. Modulation of gut 
microbiota during probiotic-mediated attenuation of metabolic syndrome in high fat diet-fed mice. The ISME J., 9: 1-15. https://doi.org/10.1038/ ismej.2014.99

Wang, X., Yu, H., Shan, A.S., Jin, Y. C., Fang, H.T., Zhao, Y., Shen, J., Zhou, C., Zhou, Y.F., Fu, Y.R., Wang, J.M. and Zhang. J., 2018. Toxic effects of zearalenone on intestinal microflora and intestinal mucosal immunity in mice. Fd. Agric. Immunol., 29: 1002-1011. https://doi.org/10.1080/09540105. 2018.1503233
Wild, C.P. and Gong, Y.Y., 2010. Mycotoxins and human disease: a largely ignored global health issue. Carcinogenesis, 31: 71-82. https://doi.org/10.1093/ carcin/bgp264

Zinedine, A., Soriano, J.M., Moltó, J.C., and Manes. J., 2007. Review on the toxicity, occurrence, metabolism, detoxification, regulations and intake of zearalenone: an oestrogenic mycotoxin. $F d$. Chem. Toxicol., 45: 1-18. https://doi.org/10.1016/j. fct.2006.07.030 